

\section{ÍNDICE}

\section{ÁMBITOS PERSONALES PERSONAL ÁMBITOS}

Crisis de Venezuela: Análisis y perspectivas según los titulares de la prensa argentina, española y china

Venezuela's crisis: Analysis and perspectives in the headlines of Argentine, Spain and China press

Hui Feng Liu

Tratamiento informativo de la violencia de género: asesinatos de mujeres. Análisis de la agencia EFE

Informative treatment of gender violence: murders of women. Analysis of the press agency EFE Rosa Rodríguez Cárcela, Agustín López Vivas

MONOGRAFICOS MONOGRAPHS

Presentación Monográfico. El universo transmedia de los medios de comunicación universitarios: acción dentro y fuera del aula en la sociedad postdigital

Antonia Isabel Nogales-Bocio, Ángels Álvarez villa

El papel transformador de la radio universitaria en materias teóricas ajenas a la comunicación

The transformative role of college radio in theoretical subjects outside communication

Miguel Ángel Díaz Monsalvo

La radiodifusión universitaria: acción discursiva radiofónica para la divulgación de la ciencia University Radio Broadcasting: Radiophonic Discursive Action for the Science Popularization Jorge Sadi Durón, Joel Zapata Salazar

El uso corporativo de Instagram en las universidades privadas españolas. Estudio comparativo de treinta y cinco universidades

The corporate use of Instagram in spanish private universities. Comparative analysis of thirty-five private universities 
La radio universitaria como herramienta de inclusión social: OndaCampus en contextos como la cárcel y barrios desfavorecidos

The university radio as a tool for social inclusion: OndaCampus in contexts like the prison and disadvantaged neighborhoods

Leonor Real Adame, Daniel Martín-Pena, Macarena Parejo Cuéllar

Hacer radio universitaria en la era de YouTube: uso de la plataforma de vídeos a demanda en el contexto mexicano

Make college radio in the age YouTube: use of video on demand platform in the Mexican context Marina Vázquez Guerrero

\section{ARTÍCULOS ARTICLES}

O impresso e o digital nos modelos de negócios de jornais locais: uma análise do Sermos Galiza

The press and digital in business models from local media: Analysis of newspaper Sermos Galiza

Giovanni Ramos

Publicidad y cáncer en la prensa escrita (1903-1912)

Advertising and cancer in the written press (1903-1912)

Laura Almudéver-Campo, Ramón Camaño-Puig

Game rules vs. fandom. How Nintendo's Animal Crossing fan-made content negotiates the videogame meanings

Las reglas del juego vs. el fandom. Cómo el contenido hecho por fans de Nintendo Animal Crossing negocia los significados del videojuego

Jose A. Moreno

La comedia de situación y su análisis textual: evolución de los elementos constructivos del formato

The sitcom and its textual analysis: evolution of the constructive elements of the format

Darío Martín Sánchez

Análisis del discurso emocional de Donald Trump en la campaña electoral de $\mathbf{2 0 1 6}$ Analysis of Donald Trump's emotional speech on the 2016 election campaign 
Innovar, comunicar y transformar (en) la Universidad

Innovate, communicate and transform (at) the University

María Sofía Bernat

288-292

Transparencia en los medios: Un requisito imprescindible para medir la rentabilidad social en radio y televisión

Transparency in the media: An essential requirement to measuresocial profitability in radio and television

Amanda Salazar

Los estudios feministas en comunicación: representación de las mujeres en la revolución tecnológica

Feminist studies in communication: representation of women in the technological revolution 


\title{
Hacer radio universitaria en la era de YouTube: uso de la plataforma de vídeos a demanda en el contexto mexicano
}

\author{
Make college radio in the age YouTube: use of video on demand \\ platform in the Mexican context
}

\author{
Marina Vázquez Guerrero, Universidad de Colima, \\ Av. Universidad 333, colonia Las Víboras, Colima, México \\ marina@ucol.mx | Orcid: https://orcid.org/0000-0001-9875-4211
}

DOI: http://dx.doi.org/10.12795/Ambitos.2020.i47.08

\section{Resumen}

En el presente artículo se busca reflexionar sobre las estrategias de comunicación digital que actualmente utilizan las radios universitarias mexicanas, que les permite acercarse y ampliar una audiencia, que avanza rápidamente hacia el consumo en línea. De manera específica, el estudio se enfoca sobre el uso de la plataforma de vídeos a demanda, YouTube que, dado el alcance y su fácil acceso en los smartphones, significa una herramienta indispensable para estar presente en la cultura digital de personas jóvenes, que se ha comprobado, prefieren lo audiovisual. A través del análisis de contenido a los canales de vídeo de las ocho radios universitarias del país que lo utilizan, los resultados muestran una baja participación en general; un uso limitado a eventos masivos y algunos programas en vivo. En los mejores casos se observa la unión de esfuerzos con las televisoras de la misma institución y la creación de series cortas para promover artistas o temas literarios. Los contenidos en su mayoría son de tipo cultural y de interés social. La discusión final se centra en abundar sobre las oportunidades que están dejando de lado estos medios de corte social al no 
incorporar estrategias interactivas en el segundo buscador más grande del mundo, con el que se podría visibilizar y difundir aún más la labor cultural y divulgativa de la radio universitaria, cuya prioridad está en la ciencia, cultura y educación.

\begin{abstract}
This article analyzes the digital communication strategies currently used by Mexican university radios. The purpose of these strategies is to help university radio stations throughout Mexico to approach and expand their audiences, who are rapidly turning towards online consumption for information and entertainment. Specifically, this study focuses on the use of the video-on-demand platform, YouTube. Due to YouTube's scope and easy access on smartphones, it has become an indispensable tool in the digital culture of young people, who have been proven to prefer audiovisual content (add reference to the study that tested this). This study analyzed the content of all eight Mexican university radio stations that offer video channels. The results show low overall participation, as well as limited use for mass events and live programs. Some institutions have found better success rates by partnering with the university's television station to create short series promoting artists or literary themes. The majority of content produced is cultural or social. The final discussion focuses on the opportunities that are being neglected by not incorporating interactive social media strategies into University radio stations, whose priorities are spreading information about science, culture and education to their respective audiences.
\end{abstract}

Palabras clave: Radio educativa, radio universitaria, comunicación, juventud, redes sociales.

Keywords: Educational radio; university radio, communication, Youth, social media.

\title{
1. INTRODUCCIÓN
}

La comunicación pública es un derecho de toda sociedad democrática. En aquellos contextos donde prime el poder del mensaje comercial en el ecosistema mediático, se está viviendo en una realidad desequilibrada, marcada por el consumismo. Varios autores han advertido sobre lo que nos ha dejado el capitalismo globalizado, que promueve productos y estilos de vida estéticos y masivos a nivel global (Lipovesky y Serroy, 2016) y por lo tanto, dejando en la sociedad un pensamiento único (Ramonet, 1998).

En este contexto, la radio universitaria forma parte de ese grupo de medios no lucrativos, que hacen un contrapeso a la visión mercantilista de la época. Allí aparecen las radios públicas, comunitarias, sociales, educativas y escolares de las que por los años ochenta mencionaba MacBrian (1980), era necesario acrecentar porque la concentración mediática era alta. 
A más de 35 años de este documento que movió la opinión pública, académica y política de la época, se ha podido constatar que la concentración de medios en el mundo no ha decrecido, al contrario, con la implementación de Internet y los servicios de telefonía móvil y digital existen grandes emporios comunicativos que influyen de manera importante en los contenidos de los consumidores en todo el mundo. (Vázquez Guerrero y Martín Pena, 2017, p. 140)

Respaldadas por instituciones educativas, las radios universitarias, en su mayoría de carácter público, luchan contra esa corriente dominante que, cobijada por las nuevas tecnologías, tomó más fuerza a nivel mundial.

$Y$ es que desde hace casi cien años que las universidades hicieron uso de los micrófonos para emitir programas con contenidos en otro sentido al modelo de entretenimiento que dominó en casi todo el mundo, ha habido un desarrollo importante divulgando la cultura, la ciencia, los temas académicos y sociales. Las pioneras radios universitarias tuvieron épocas gloriosas, aunque nunca especialmente masivas y sin embargo, hoy siguen presentes con nuevos modelos y formatos, buscando la permanencia.

La era de Internet le ha dado más visibilidad y ha permitido su crecimiento, logrando experiencias novedosas como en el contexto español (Casajús y Martín-Pena, 2018) donde a pesar de no existir una ley que las respalde, siguen apareciendo, demostrando su pertinencia para la formación de estudiantes y la difusión de la cultura.

En el caso específico de México, existe una amplia oferta de radios a partir de 1937, fecha en que se inauguró la señal de Radio UNAM, emisora de la máxima casa de estudios. La mayoría de las universidades estatales más importantes cuentan con una radio - San Luis Potosí, Veracruz, Guanajuato, Oaxaca, Sonora, Yucatán, Baja California, Hidalgo, Morelos, Querétaro, etc.-, y obtuvieron un permiso de antena con una cobertura no siempre amplia pero significativa. Poco a poco se fueron sumando hasta el punto de que en 2005 se reportaban 65 emisoras en 26 estados de la república (Reyna, 2004). Con la llegada de Internet aparecieron además, las radios online que ampliaron la oferta, sobre todo de centros educativos privados y tecnológicos hasta sumar más de 75 que mantienen la señal por más de 85 frecuencias (Vázquez y Chamizo, 2014).

Diferentes estudios previos (Vázquez Guerrero, 2012; 2015) advierten sobre la necesidad de lograr mayor vinculación juvenil y tecnológica con el uso de formatos híbridos que permitan su difusión por otros medios. El consumo juvenil es bajo, pero el acercamiento a ellos permitió saber en un contexto específico que "es posible mejorar los porcentajes de audiencia estudiantil si la radio busca estrategias de acercamiento 
físico y les da opciones en la web 2.0" (Vázquez Guerrero; García Lezama; López Chagoya y Velasco, 2016, p. 79).

Por medio del presente texto, se buscará observar de qué manera la radio universitaria mexicana participa en la adopción de plataformas digitales que permitan ampliar su audiencia, en particular hacia aquellas generaciones con una gran cultura audiovisual, que pasan más de 8 horas al día conectados a Internet (AMIPCI, 2019); haciendo un consumo creciente a través del Smartphone y que no han sido el público mayoritario de las radios universitarias clásicas.

Si bien Facebook se ha convertido en la plataforma que superó la transmisión online y las páginas web en el contexto mexicano (Vázquez Guerrero, 2019), ahora se busca revisar de qué manera se recurre a otras plataformas que son igualmente populares entre las personas y que tienen compatibilidad con medios como la radio.

Por lo tanto en este artículo se busca plantear un enfoque específico con el siguiente objetivo general:

Identificar el uso que se le da a la plataforma de vídeos a demanda YouTube en las radios universitarias de México.

De manera específica se buscó:

- Conocer las características de los canales de YouTube de las emisoras universitarias de México.

- Revisar el tipo de formatos y de contenido de las publicaciones de las emisoras universitarias de México que cuentan con YouTube.

- Confirmar la idoneidad del uso de la plataforma YouTube como estrategia de acercamiento para las nuevas generaciones en las emisoras universitarias de México.

\subsection{La era YouTube}

YouTube fue el invento del año en 2006 porque consiguió una verdadera revolución en la joven red de redes: permitió el acceso de muchos usuarios a subir y consumir contenidos de su interés, sin tener que pagar por ello, haciendo posible "el acto de 'compartir' vídeos online" (Van Dijk, 2016), sin duda algo inédito. Con esa dinámica rompió el concepto de aldea global de McLuhan al lograr la primera experiencia masiva de vídeo on demand (Scolari, 2008) que se ha mantenido creciente desde entonces. Si bien se dice que una vez que pasó a manos de Google, la plataforma perdió su esencia porque su originalidad radicaba en que la hizo crecer una comunidad creativa ( Van Dijk, 2016), no cabe duda de que se ha ido complejizando y dando diferentes opciones de uso como el live streaming, el programa de 
monetización para creadores y youtubers; su canal de música, de niños y la renovada versión sin anuncios -YouTube Premium-, generando una economía alrededor del entretenimiento que ha sido difícil de igualar por otras plataformas.

Quienes participan como creadores audiovisuales en esta comunidad virtual han aportado también un lenguaje propio con mensajes que muestran un estilo de acuerdo con los códigos de la red. Como afirma Quintero (2017), "se ajustan principalmente hacia lo fragmentario y lo hipertextual. El autor recursivo insinúa la directriz de un mensaje fragmentado y la comunidad de usuarios interviene en la construcción y fortalecimiento del mismo" ( p. 89).

La plataforma de YouTube ha sido estudiada y recomendada como un recurso importante para la enseñanza y aprendizaje en diferentes disciplinas (Duffy, 2008; Burke, Snyder y Rager, 2009) y actualmente es una fuente importante de consulta de estudiantes y aprendices de todo tipo de saberes y oficios, sobre todo por su acceso libre y de bajo costo (Pardo Abril, 2008).

Al mismo tiempo estamos hablando de una empresa que lucra, que de alguna manera controla y tiene poder y que forma parte del monopolio tecnológico de una empresa global que dicta la agenda de tendencias con ayuda de los usuarios, quienes viralizan contenidos, no siempre sanos, en cuestión de segundos. A pesar de sus normas éticas contra material obsceno, peligroso e inadecuado y su política de protección a los derechos de autor, sigue siendo polémica y a la vez un repositorio infinito de conocimiento.

Actualmente la empresa cuenta con versiones para 91 países en 80 idiomas y la dinámica de consumo es de mil millones de horas de reproducción diarias con más de mil millones de usuarios, de acuerdo con datos directos de YouTube ${ }^{1}$. Su presencia, sin embargo, es inevitable en este momento histórico en que es líder y del cual pueden obtener beneficios las organizaciones no lucrativas como las radios universitarias, sin caer en la zona superficial del portal. La prensa internacional, por ejemplo, ha sabido aprovechar este recurso, en vista del abandono de sus lectores. Diarios como El País y El Mundo en España y Milenio en México, son un gran ejemplo. Aparecen en la lista de los 250 portales de noticias más importantes en YouTube (Socialblade, 2019).

Como afirma Jenkins (2017, párr. 8) "En la era de YouTube, las redes sociales emergen como una de las habilidades sociales y culturales más importantes que los jóvenes necesitan adquirir si van a convertirse en participantes significativos en la cultura que los rodea".

Por otra parte, México, con una población de casi 120 millones de personas aún no logra dar acceso a Internet a todos los sectores sociales. Actualmente alrededor del 
$66 \%$ (INEGI, 2019) lo utilizan y hacen un consumo alto de redes sociales como Facebook (98\%); WhatsApp (91\%) YouTube (82\%) e Instagram (57\%) (IAB, 2019).

El interés por revisar el uso que se hace de YouTube tiene que ver no sólo con el crecimiento entre los potenciales usuarios de la radio universitaria, sino porque la tendencia en el país es que el $91 \%$ de las personas busca información en vídeo y sus intereses de contenido tienen relación con tecnología (55\%) entretenimiento (50\%) deportes $(48 \%)$ y noticias $(45 \%)$ (IAB, 2018), mismos que pueden propiciar las instituciones educativas. Además, otro estudio manifiesta que YouTube es la plataforma más utilizada para ver contenido en Internet en México y la acción de pagar suscripción se ha dado de manera amplia solamente con Netflix, que era consumido al momento del estudio por sólo el 25\%, muy lejos del uso de YouTube (IFT, 2018).

Por otra parte, los datos de consumo de radio por Internet en México no son muy numerosos, del $22 \%$ de las personas que declararon escuchar radio, el $38 \%$ lo hace por la red de redes. Sin embargo, cada vez son más las personas que escuchan contenidos de audio o música por esa vía, en un 35\% de la población (IFT, 2018).

Es decir, hay un público que escucha en línea, pero es aún más relevante el uso de algún soporte audiovisual para obtener más atención, dado que hay un interés creciente por el vídeo en línea que incluye series, tutoriales, vídeos instructivos y conciertos, enlazados o promovidos por medio de redes sociales (IAB,2018).

\section{APROXIMACIÓN TEÓRICA}

Con la ayuda de autores que son afines a la adopción de la tecnología para el desarrollo social y la ecología de medios, así como a quienes advierten de las consecuencias de la homogeneización del capitalismo artístico, plantéo la siguiente mirada al fenómeno de la radio e Internet.

Hacia 2008, Scolari ya apuntaba la existencia de las hipermediaciones, entendidas como "procesos de intercambio, producción y consumo simbólico que se desarrollan en un entorno caracterizado por una gran cantidad de sujetos, medios y lenguajes interconectados tecnológicamente de manera reticular entre sí" (Scolari, 2018, p.114), que habían modificado todo el sistema comunicacional.

La comunicación de masas, que usaba tecnología analógica se transformó para dejar su propio lenguaje y combinarse para participar de un nuevo diálogo presente en los entornos multimedia que tienen como característica la hipertextualidad, la multimedialidad y la interactividad.

Allí se observaba cómo los jóvenes ya contaban con nuevas competencias a partir de sus experiencias de consumo hipertextual y sus intereses eran más audiovisuales; 
personalizados y cercanos a la experiencia de vídeo on demand, por lo que es necesario que los medios tradicionales - como la radio-, adapten sus contenidos al nuevo consumidor que ha dejado de ser audiencia, de connotación pasiva, a usuarios "marcados por la personalización de los contenidos, el consumo asincrónico y el intercambio muchos-a-muchos" ( Scolari, 2008, p. 288).

Por otra parte, Castells (2014) afirma que todo cambio tecnológico importante genera "una mitología propia", en gran medida porque lo usamos previo a que la ciencia revise sus efectos y lo que involucra. Sin embargo, es inevitable que vivimos en una sociedad red que tiene como base las redes personales a nivel global "resultado de la interacción entre el paradigma tecnológico emergente basado en la revolución digital y determinados cambios socioculturales de gran calado" (p. 12).

Martín-Pena (2013) apunta que gracias a las tecnologías de información y comunicación se mejoraron los procesos más importantes de la radio al punto de que "hace uso de una plataforma multimedia, en la que el sonido ya no es el único actor principal, ahora convive con el texto, la imagen fija y en movimiento. La radio se vuelve híbrida, se enriquece y su dial tiende al infinito" (p. 180).

Internet nos lleva ahora a procurar no sólo participar de la comunicación de manera digital sino de propiciar la interactividad, que caracteriza a muchas de las herramientas actuales. La radio en línea es parte del esquema de uno a muchos, sin embargo Twitter, el Podcasting, Flick o los blogs forman parte de la comunicación monomedial pero donde se logra la conversación de muchos a muchos. De acuerdo con Scolari (2008), plataformas como YouTube cuenta con este tipo de conversación, además de la multimedialidad que lo hace una herramienta más completa y a la vez compleja.

En el caso específico de YouTube, que como se mencionó líneas arriba, es una plataforma muy consultada en la sociedad mexicana, ya forma parte de la cultura visual de una sociedad, que tiene más de un siglo de consumirla en formatos convencionales como el cine o el video. "El universo YouTube es tan amplio como inabarcable. No solo en cuanto a la diversidad y cantidad de material audiovisual que almacena, sino en relación con las opciones y posibilidades que les brinda a sus usuarios" (Murolo y Lacorte, 2015, p. 26).

Finalmente cabe aclarar que la recomendación de incorporación el uso de esta plataforma en las radios universitarias se propone principalmente para contribuir en la difusión de sus programas en primera instancia, pues la radio con señal en directo tiene su personalidad propia para seguir siendo la protagonista; sin embargo, en las radios online y con podcast, es una herramienta cotidiana importante. También se recomienda en el uso de actividades puntuales como transmisión de programas, de eventos relevantes y en la creación de contenidos específicos para que la audiencia juvenil se interese por la radio. No se trata de entrar en la dinámica de saturación de 
información y medición masiva de audiencia que manejan las empresas mediáticas comerciales sino de recurrir al portal para participar de una cultura audiovisual que domina.

En estudio previo se observaba que las universidades iberoamericanas utilizan la plataforma con tres fines que las autoras Guzmán y del Moral (2014) dividen en las siguientes categorías: “1) Institucionales: promoción de los servicios, 2) Académicas: interacción con la comunidad educativa; y, 3) Comunicativas: invitación a participar en actividades" (p. 89). Esta práctica se extiende en pocos casos a sus medios, por lo que se busca hacer esta revisión.

\section{METODOLOGÍA}

A través de una mirada reflexiva sobre la técnica del análisis de contenido, se aborda este estudio, considerando los datos previos de la observación sistemática de 71 sitios web oficiales de las radios universitarias mexicanas (Vázquez Guerrero, 2019), en las que se registró el tipo de herramientas de comunicación digital que utilizaban. Allí se obtuvo el dato de las 12 emisoras que contaban con canal de YouTube, para aplicar un análisis con mayor énfasis en lo cualitativo.

Al final la muestra se conformó por las ocho emisoras que tenían actividad reciente de su canal, representando tan solo el $11 \%$ de las radios existentes en el país, pues se detectó que las otras cuatro no le daban uso frecuente. De allí la determinación de estudiar solamente las que tenían actividad en el momento de la recolección de datos. Son seis de universidades públicas y dos de privadas. Cinco ubicadas en el centro del país; dos en el centro-occidente y una en el norte. 
Tabla 1

Muestra de canales de YouTube de las radios universitarias analizadas

\begin{tabular}{|c|c|c|}
\hline No. & Nombre de emisora & Universidad \\
\hline 1 & Concepto Radial & ITESM, campus ciudad de México (privada) \\
\hline 2 & Ibero 90.9 & $\begin{array}{c}\text { Universidad Iberoamericana, campus Santa Fe, ciudad } \\
\text { de México (privada) }\end{array}$ \\
\hline 3 & Radio Chapingo & $\begin{array}{l}\text { Universidad Autónoma Chapingo, estado de México } \\
\text { (pública) }\end{array}$ \\
\hline 4 & Radio UdeG Ocotlán & Universidad de Guadalajara, Ocotlán, Jalisco (pública) \\
\hline 5 & Radio UNAM & $\begin{array}{l}\text { Universidad Nacional Autónoma de México, ciudad de } \\
\text { México (pública) }\end{array}$ \\
\hline 6 & UACJ Radio & $\begin{array}{l}\text { Universidad Autónoma de Ciudad Juárez, Chihuahua } \\
\qquad \text { (pública) }\end{array}$ \\
\hline 7 & Uni Radio & $\begin{array}{l}\text { Universidad Autónoma del Estado de México, Toluca } \\
\qquad \text { (pública) }\end{array}$ \\
\hline 8 & Universo 94.9 & Universidad de Colima, Colima ( pública) \\
\hline
\end{tabular}

Fuente: Elaboración propia.

- En cuanto a las variables revisadas se encuentran:

- El número de suscriptores

- Número de vistas

- Número de publicaciones

- La frecuencia de publicación dividida en diarias, semanales, quincenales, irregulares o solo en eventos.

De forma cuantitativa como cualitativa se observó además el tipo de formatos y de contenido publicado, en las subcategorías que se muestran en la tabla 2 : 


\section{Tabla 2}

Tipos de formatos y de contenidos analizados en los canales de YouTube de las emisoras universitarias

\begin{tabular}{|l|l|}
\hline \multicolumn{1}{|c|}{ TIPO DE FORMATOS } & \multicolumn{1}{c|}{ TIPO DE CONTENIDO } \\
\hline Programa en vivo & Ciencia \\
\hline Evento en vivo & Cultura \\
\hline Vídeo editado & De interés social \\
\hline Anuncio o spot & Artístico \\
\hline \multirow{3}{*}{ Otro } & Entretenimiento \\
\cline { 2 - 2 } & Académico \\
\cline { 2 - 2 } & Institucional \\
\cline { 2 - 2 } & Oficial (gobernantes, rectores) \\
\cline { 2 - 2 } & Otro (especificar) \\
\hline
\end{tabular}

Fuente: Elaboración propia.

La temporalidad del estudio para la primera etapa fue de febrero de 2018; para la segunda etapa, abarca la trayectoria general de los canales y en la parte cualitativa se analizaron las últimas 20 publicaciones realizadas en YouTube, hasta el 15 de marzo de 2019.

Para la obtención de datos complementarios que permitieron verificar de manera global la funcionalidad de los canales, se recurrió la aplicación digital Competitor comparison tool ${ }^{2}$, utilizada con mayor frecuencia para estudios de mercadotecnia.

\section{RESULTADOS}

\subsection{Características de los canales de YouTube de las radios universitarias}

Los resultados nos muestran que en México hay una baja participación de radios que cuentan con canal de YouTube, sin embargo, las que lo tienen, realizan esfuerzos diversos que vislumbran la pertinencia de su uso, si se tuviera una planeación estratégica integrada.

El primer hallazgo general es que, de las 8 radios, 4 habían bajado el ritmo de publicación a fines de 2018 y las otras 4 continuaban haciéndolo. Es decir, que existe la posibilidad de que alguna radio deje de publicar por largas temporadas y que sea parte de lo cotidiano, de acuerdo con sus prioridades.

Para dar cuenta del número de suscriptores, en la tabla 3 se muestran los canales con sus respectivos seguidores a marzo de 2019. Aquí aparece como líder el canal de la Universidad Iberoamericana con casi 5,500 suscriptores a la fecha. 
Tabla 3

Seguidores por emisora

\begin{tabular}{|l|l|l|}
\hline No. & \multicolumn{1}{|c|}{ Emisora } & \multicolumn{1}{c|}{ Seguidores } \\
\hline 1 & Ibero 90.9 & 5,408 \\
\hline 2 & UACJ Radio & 3,328 \\
\hline 3 & Radio Chapingo & 2,283 \\
\hline 4 & Radio UNAM & 2,001 \\
\hline 5 & Uni Radio & 1,420 \\
\hline 6 & Radio UdeG Ocotlán & 535 \\
\hline 7 & Concepto Radial & 131 \\
\hline 8 & Universo 94.9 & 86 \\
\hline
\end{tabular}

Fuente: Elaboración propia con datos de canales oficiales de YouTube de las emisoras.

Por otra parte, en la figura 2 se describe comparativamente su crecimiento de un año a otro. Aquí destaca la radio de la Autónoma de Ciudad Juárez que tuvo un crecimiento de más del 100 por ciento, de 1,602 a 3,328 en un año.

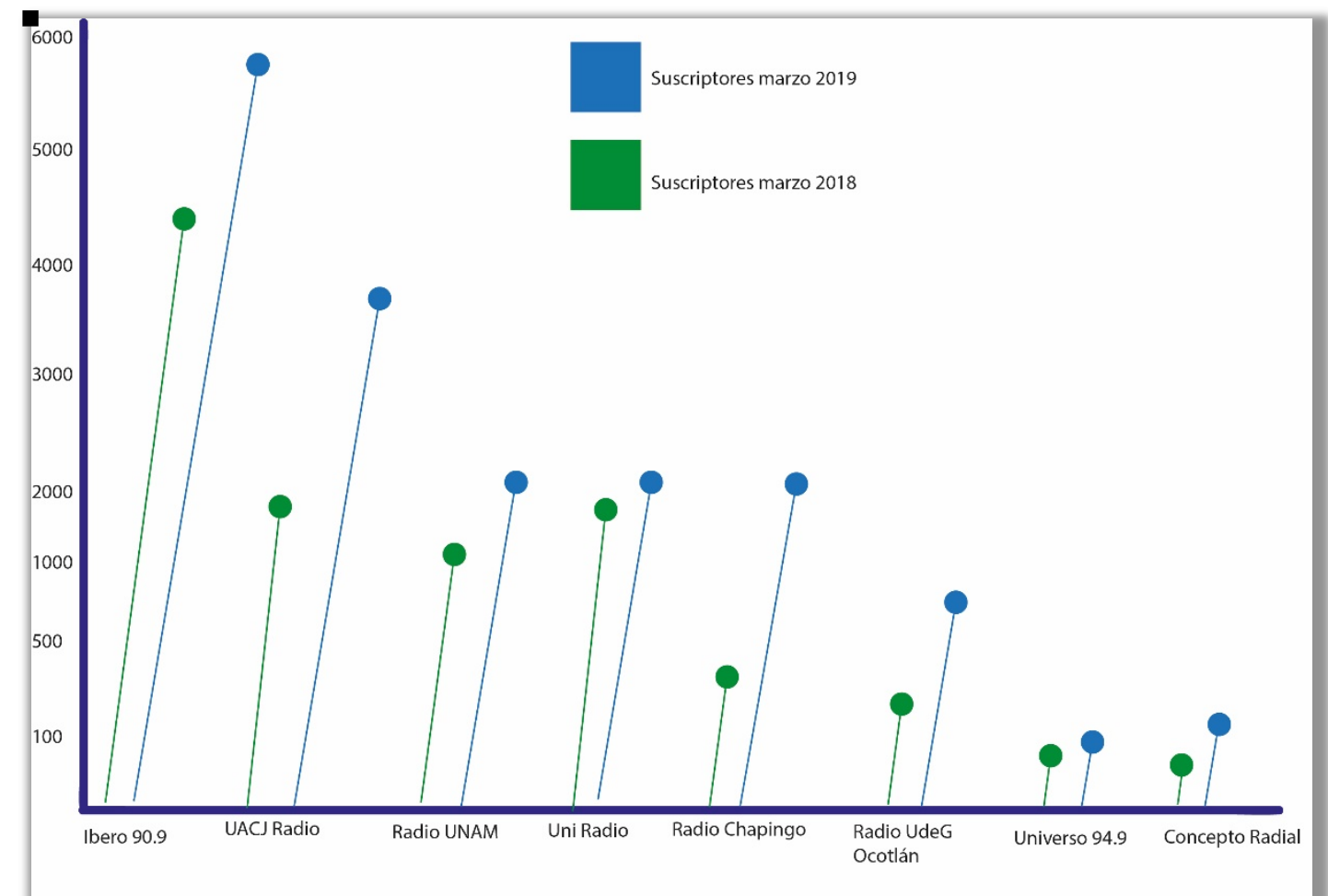

Figura 1. Crecimiento comparativo entre las radios universitarias con YouTube Fuente: Canales oficiales de YouTube de las radios analizadas. 
La radio universitaria de Chapingo que tenía pocos suscriptores en 2018 multiplicó su audiencia en un $420 \%$ de 444 a 2,283 seguidores. Otro caso relevante fue Radio UNAM que creció en más del $62 \%$ respecto a sus cifras ya en aumento. Por su parte Concepto radial aumentó en un $118 \%$ sus seguidores en el transcurso de un año, dejando atrás a Universo que sólo aumentó $6 \%$.

Otro dato relevante en el uso de la plataforma de YouTube es el número de vistas que obtienen las publicaciones (tabla 4). UACJ Radio es la radio que lidera con 849,239 vistas. Su más cercana seguidora, Ibero 90.9 , tiene una gran desventaja con 488,816 y por lo consiguiente con la tercera, Radio Chapingo que cuenta con 260,851 vistas. La radio que tiene menos movimiento es Uni Radio con 6,704 vistas, lo que la hace poco interesante para el usuario.

\section{Tabla 4}

Emisoras con canal de YouTube y cifras relevantes

\begin{tabular}{|c|c|c|c|}
\hline No. & Nombre de emisora & Vistas $^{3}$ & Publicaciones $^{4}$ \\
\hline 1 & Ibero 90.9 & 488,816 & 316 \\
\hline 2 & UACJ Radio & 849,239 & 2901 \\
\hline 3 & Radio UNAM & 47,806 & 111 \\
\hline 4 & Uni Radio & 6,704 & 24 \\
\hline 5 & Radio Chapingo & 260,851 & 318 \\
\hline 6 & Radio UdeG Ocotlán & 131,949 & 749 \\
\hline 7 & Universo 94.9 & 18,740 & 82 \\
\hline 8 & Concepto Radial & 58,118 & 67 \\
\hline
\end{tabular}

Fuente: Canales oficiales de YouTube de las radios analizadas, a partir del análisis realizado a través de Competitor Comparison Tool.

El número de publicaciones fue otra variable a observar en el mundo de la interacción digital y no siempre corresponde con las anteriores cifras de suscriptores y viewers. En 
esta muestra, la radio que tiene más actividad publicando es precisamente UACJ Radio con más de 2,900. En segundo sitio, pero lejano está Radio UdeG Ocotlán con 749 .

Una de las razones por las que esta muestra es variable tiene que ver con la frecuencia de publicaciones. Hay quienes tienen muchas pero su ritmo trabajo no es claro. sólo la Radio UdeG Ocotlán tiene un plan fijo semanal; seis, que significan el $75 \%$, son irregulares al no observar en ellas un ritmo de publicación y una más Radio UNAM-, publica sólo en eventos, lo que muestra poco aprovechamiento del medio.

En ese sentido cabe destacar que la radio pionera en el uso de la red social es precisamente Ibero 90.9 que desde 2007 la utiliza, es la de más suscriptores, pero quien más publicaciones y visualizaciones realiza es la productora de la Universidad Autónoma de Ciudad Juárez quien ha sabido hacer un buen uso del recurso, dado que no tiene una emisora para dar a conocer sus productos y comparte el canal con los contenidos televisivos de la universidad.

Las cifras de estas radios, sin embargo, no son suficientes, para lograr un buen posicionamiento, ya que de acuerdo con Competitor comparison tool, el engagement logrado a través del análisis de sus vistas, likes, dislikes, material compartido y seguidores por estas emisoras, es muy bajo. Ibero 90.9 tiene 69.7 puntos de 100 y es la mejor evaluada de las ocho.

\subsubsection{Los formatos}

En la radio universitaria los formatos audiovisuales para YouTube son limitados. En cuanto a la práctica, lo más común es el vídeo editado en 7 de los 8 canales. Le sigue el anuncio o spot en 5, el evento en vivo en 4 y la transmisión de programas en vivo en 3 de estos canales. Lo que es común en esas transmisiones en vivo son las actividades de corte académico, institucional, deportivo o cultural como las ferias y festivales. Los vídeos cortos con edición y producción son pocos: Uniradio, Universo 94.9 y Concepto radial son los que tienen antecedentes de proyectos interesantes en ese sentido, pero sin continuidad. Uniradio cuenta con una serie corta de recomendaciones de libros y discos realizada por los colaboradores de la emisora, con un poco de producción y buen ingenio. Universo, destaca contenido cultural con una serie titulada el Poder de la poesía con el breve testimonio de escritores colimenses; producción sencilla y agradable, así como algunos formatos experimentales para conmemorar la revolución mexicana o para dar a conocer el tema de un programa humorístico. Por su parte, Concepto radial muestra una tendencia a las temáticas de tipo científico, cultural y de interés social con vídeos sobre fechas relevantes en la historia, entrevistas con académicos o cápsulas. 


\subsubsection{El contenido}

De los canales analizados, en términos generales lo que más se encontró de contenido fueron los temas relacionados a las diferentes manifestaciones culturales en todos los canales $(29 \%)$ seguidas de los de interés social $(25 \%)$ y académico $(15 \%)$. Sin embargo, las radios de UACJ, Chapingo y UdeG son los que más transmiten temas institucionales y oficiales, con un $7 \%$ y $6 \%$ respectivamente. Los temas científicos son pocos: De toda la muestra Concepto radial es la única que tiene un espacio (6\%). El entretenimiento también es abordado en menor medida (5\%) al igual que lo artístico $(4 \%)$.

Este estudio da cuenta de la forma en que cada radio refleja su personalidad y temas prioritarios en la plataforma. Muestran una cara del perfil de la Universidad a la que pertenecen. Por tanto, es interesante observar que la mayoría busca presentar contenidos complementarios, de corte cultural y social y otras atienden los asuntos oficiales y de interés hacia la comunidad como las sesiones de Consejo Técnico y comunicados ante huelgas, que vivían por ese momento algunas universidades.

Respecto a la calidad de los contenidos destaca Ibero 90.9 y Radio UNAM siendo coherentes con lo que emiten por antena y por las redes sociales, no obstante, la primera había dejado de publicar por esa vía, dando prioridad a otras redes sociales y la segunda la usa sólo en eventos y no le hace promoción en su página web.

\section{CONCLUSIONES Y DISCUSIÓN}

Como afirma López Villafranca (2017) en su análisis a las redes sociales de programas de radio en España, estas plataformas "se consolidan como el principal medio para difundir los contenidos de los programas, hacerlos virales, llegar a otros públicos que no son los oyentes habituales y lograr captar audiencia que sólo consume estos espacios en internet" (p. 1875), esta podría ser la idea de quienes desde una radio universitaria participen de las ventajas de la red. Quizá no se logre llegar a tener un $100 \%$ de engagement como lo buscarían los medios comerciales, pero la presencia de una manera ordenada y firme ya es relevante.

Otros ejemplos de uso exitoso se han dado en el ámbito informativo que ha permitido refrescar formatos, ampliar audiencias e incluso mejorar su presencia o imagen de marca (Ruvio Manzano y Gomes-Franco e Silva, 2019). En el caso de las emisoras universitarias, la imagen institucional tendría una presencia importante entre las comunidades virtuales.

Respecto a los objetivos planteados en el estudio, podemos ver en primer lugar que las características de los canales de YouTube de las ocho emisoras universitarias que lo tienen no hacen un uso eficiente desde el momento mismo de no tener una 
periodicidad de uso. Se alcanza a leer entre líneas una falta de estrategias explícitas que hacen ver a los canales con poca actividad. Se observa un uso limitado a eventos masivos y algunos programas en vivo o la unión de esfuerzos con las televisoras de la misma institución, en el mejor de los casos. Sin embargo, esos breves esfuerzos, dan fe de la posibilidad de incluir estas prácticas a otros proyectos radiofónicos universitarios.

Los contenidos en su mayoría son de tipo cultural y de interés social, cuando la carta programática de la mayoría es más amplia y diversa. Tampoco se observa en la mayoría, un interés genuino por vincular a los jóvenes, a los futuros radioescuchas o nuevas generaciones que hacen un consumo audiovisual de manera natural por ser parte de los nativos digitales. Esto significa que se está desaprovechando un recurso online que tiene bajo costo de mantenimiento y producción, y que perfectamente puede converger con los contenidos comerciales que abundan y saturan la lista de intereses juveniles en la red.

Se confirma, desde el punto de vista de la autora, que esta red, identificada con contenido de entretenimiento, es idónea para ser utilizada por las radios universitarias mexicanas por diferentes razones: 1) su temporalidad, que permite la permanencia en el tiempo; 2) su formato, pues se pueden abordar formatos de acuerdo a las características multimodales de los hipermedios que son cortos, dinámicos, frescos e interactivos y la posibilidad de incluir más 3) contenidos, relacionados a la ciencia, las artes, los problemas sociales y de interés académico que requiere no sólo la comunidad universitaria para la que trabajan.

Son necesarias también las estrategias de acercamiento para las nuevas generaciones, a quienes vale la pena recordarles el papel social y cultural que tiene una radio universitaria, cuyo objetivo primordial es la extensión de la cultura y la promoción de la educación, pero también con aportar valores y contribuir a mejorar la calidad de vida de la sociedad para la que se deben. Considerar a la hora de planear las estrategias digitales de un medio universitario, en su contribución imprescindible contra la dinámica que traen cientos de empresas mediáticas trasnacionales que "sirven más para el condicionamiento y el cerco de los ciudadanos que para su emancipación" ( Ramonet, 2007, p. 87).

Las limitaciones que tiene este ejercicio se identifican quizá en la muestra obtenida sobre las publicaciones, para la parte cualitativa, pues podría ser poca para la antigüedad del canal o haber coincidido con un periodo de baja productividad y no reflejar fielmente la dinámica de este.

Queda para posterior estudio una investigación donde se confirme si los directivos y gestores de estos espacios tienen en sus planes integrar una plataforma con estas características, que sin duda requiere de un perfil profesional que no todas las radios 
podrían tener en sus presupuestos, dada la antigüedad de algunas y las políticas educativas que regularmente, en universidades públicas, están más controladas por el cuidado de la imagen institucional.

El reto sin duda es institucional en dos sentidos: hacia el exterior en primera instancia porque de acuerdo con Gutiérrez, Islas y Arribas (2018): "La nueva ecología digital trae consigo grandes retos y oportunidades para todas las personas, empresas e instituciones. Toda tecnología instituye atractivas posibilidades y por supuesto también impone determinadas limitaciones" (p. 16) y por otra parte interno, una necesidad de apertura que aún no se logra en muchos centros educativos.

\section{Notas}

${ }^{1}$ YouTube (2019) Canal oficial. YouTube para la prensa. Recuperado de: https://www.youtube.com/intl/es-419/about/press/

${ }^{2}$ Vídeo marketing comparison tool Herramienta de Análisis https://mwpdigitalmedia.com/competitor_comparison_tool/. Aplicado el 04 de marzo de 2019.

${ }^{3}$ Se refiere a los views de cada uno de los canales desde su creación hasta el 03 de abril de 2019.

${ }^{4}$ Se refiere a los vídeos publicados desde la creación del canal hasta el 03 de abril de 2019.

\section{Referencias}

AMIPCI (2019). Asociación Mexicana de Internet. 15. Estudio sobre los hábitos de los usuarios de Internet en México 2018. Movilidad en el usuario de Internet en México Recuperado de: https://cutt.ly/te6tAou

Burke, S., Snyder, S., Rager, RC. (2009). An assessment of faculty usage of YouTube as a teaching resource. The Internet Journal of Allied Health Sciences and Practice, Volume 7 Number 1.

Casajús L. y Martín-Pena, D. (2018). Social Media in University Radio: Development in its Use and Follow-up on its Platforms. En:Gallego, J.I.; Fernández-Sande y Limón, N. Trends in Radio Research: Diversity, Innovation and Policies. Diversity, innovation and policies. Newcastle upon Tyne: Cambrigde Scholars Publishing.

Duffy, P. (2008). Engaging the YouTube Google-Eyed Generation: Strategies for Using Web 2.0 in Teaching and Learning. The Electronic Journal of e-Learning Volume 6 Issue 2, pp 119-130. Recuperado de: https://files.eric.ed.gov/fulltext/EJ1098687.pdf

Castells, M. (2014). El impacto de internet en la sociedad: una perspectiva global. 19 ensayos fundamentales sobre cómo Internet está cambiando nuestras vidas. Cambio. [En línea]. Recuperado de: https://cutt.ly/le6tDsr

IAB (2018). Estudio de live video streaming, septiembre 2018 en México. Recuperado de: https://cutt.ly/Ge6tF6b 
(2019). Estudios de consumo de medios y dispositivos entre internautas mexicanos, once ed., mayo 2019. Disponible en: https://cutt.ly/fe6tGWy

IFT (2018). Instituto Federal de Telecomunicaciones. Encuesta Nacional de Consumo de Contenidos Audiovisuales 2017. Recuperado de: https://cutt.ly/ze6tHA2

INEGI (2015). Página oficial. Disponible en: https://www.inegi.org.mx/temas/estructura/

(2019) Estadísticas a propósito del día mundial del Internet (17 de mayo) datos nacionales. Recuperado de: https://cutt.ly/ue6tKJ1

Gutiérrez, F.; Islas, O. y Arribas, A. (2018). Hacia una nueva ecología mediática. Hacia un nuevo inventario de efectos. Alas. Controversias y concurrencias latinoamericanas Vol. 10 N¹6. Recuperado de: http://sociologia-alas.org/CyCL/Vol10/03_Dossier_Art1.pdf

Guzmán, A., \& del Moral, M. (2014). Trends in use of YouTube: optimizing the strategic communication of Ibero-American universities. Observatorio (OBS*), 8(1), 69-94. Recuperado de: https://cutt.ly/de6tZsg

Jenkins, H. (2017). Nine Propositions Towards a Cultural Theory of YouTube. Confessions of an ACA-FAN [blog] Recuperado de: https://cutt.ly/re6t0tz

Lipovesky, G. y Serroy, J. (2016). La estetización del mundo. Vivir en la época del capitalismo artístico. Barcelona: Anagrama.

López Villafranca, P. (2017). Análisis de los programas virales de radio en las redes sociales en España. Cuadernos del CAC 116. Recuperado de: https://cutt.ly/Re6tX5sMartín

Pena, D. (2013). Radios universitarias en España: plataformas de comunicación interactiva y redes de colaboración. Tesis doctoral. Universidad de Huelva. Recuperado de: http://rabida.uhu.es/dspace/handle/10272/7238.

MacBride, S. et al. (1980). Un Solo mundo, Voces Múltiples. Comunicación e Información en Nuestro Tiempo. México: Fondo de Cultura Económica/UNESCO.

Murolo, N., \& Lacorte, N. (2015). De los bloopers a los youtubers. Diez años de YouTube en la cultura digital. Question, 1(45), 15-29. Recuperado de https://cutt.ly/ye6tVyQ

Pardo Abril, N.G. (2008). El discurso multimodal en YouTube. Revista Latinoamericana de Estudios del Discurso 8 (1), pp. 77-107 Recuperado de: https://cutt.ly/Je6t1TW

Quintero, D. (2017). Códigos comunicativos de la producción audiovisual masiva en YouTube. Revista Comunicación No. 15. Pp. 77-90.

Ramonet, I. (1998). El pensamiento único. En I. Ramonet, Pensamiento crítico vs. Pensamiento único. Madrid, Le Monde Diplomatique/Editorial Debate.

(2007). Pensamiento único y nuevos amos del mundo. En Chomsky, N. y Ramonet, I. Cómo nos venden la moto. Información, poder y concentración de medios. 
Barcelona: Icaria.

Reyna Ruiz, M. (2005). Radios y redes: la radio en las instituciones de educación superior. Reencuentro. Análisis de Problemas Universitarios, (44), 0.

Ruvio Manzano, F. y Gomes-Franco e Silva, F. (2019). YouTube como herramienta de refuerzo de marca para la Agencia EFE. Pilares para el éxito en plataformas de vídeos digitales. Hipertext.net, (18), 35-46. DOI:10.31009/hipertext.net.2019.118.04

Scolari, C. (2008). Hipermediaciones: elementos para una teoría de la comunicación digital interactiva. Barcelona: Gedisa.

Socialblade (2019). Top 250 YouTubers news Channels. YouTube Statistics. Recuperado de: https://cutt.ly/ae6tMm

Van Dijck, J. (2016) La cultura de la conectividad. Una historia crítica de las redes sociales. Buenos Aires. Siglo XXI editores.

Vázquez Guerrero, M. (2012). La Radio Universitaria en México y España. Estudio de la participación y formación de los jóvenes. Universidad Pompeu Fabra. Tesis doctoral. Recuperado de: https://repositori.upf.edu/handle/10230/17064

(2015). La radio universitaria en Iberoamérica: trascendencia y retos. Comunicación y Medios, (31), Pág. 151 - 170 . doi:10.5354/07191529.2015.36162.

(2019) Las radios universitarias de México y sus estrategias para comunicar la ciencia en internet. Anuario electrónico de estudios en Comunicación Social "Disertaciones" Volumen 12, Número 2 (Jul-Dic 2019).

Vázquez Guerrero, M. y Chamizo, F. (2014). La Radio Universitaria en México: diversidad y contraste. En Martín Pena, D. y Ortiz Sobrino, M.A. (comps.) Las radios Universitarias en América y Europa. Madrid: Fragua.

Vázquez Guerrero; García Lezama; López Chagoya y Velasco (2016). La radio universitaria y su papel en la dieta mediática de los estudiantes de Colima, México. En Martín-Pena; Marta-Lazo y Ortiz. Perspectivas y prospectivas de la radio universitaria en la era digital. Cuadernos Artesanos de Comunicación 113. doi: 10.4185/cac113

Vázquez Guerrero, M. y Martín-Pena, D. (2017) Ante la concentración radiofónica en España y México: promover y ampliar la radio universitaria. En Covarrubias, K. y Perazzo, P. (coords.) Memorias en extenso del II Simposio Internacional Comunicación y Cultura. Problemas y desafíos de la memoria e historia oral.

Vázquez Guerrero, M., Martín-Pena, D. y Parejo, M. (2015). La divulgación científica a través de la radio universitaria en España y México. Razón y Palabra, 19 (3-91), 669-686.

\section{Canales de YouTube analizados}


Concepto Radial https://www.youtube.com/channel/UCbzuNhtevRSiyE6vYt7x3ng/vídeos Ibero 90.9 https://www.youtube.com/user/ibero909/vídeos

Radio Chapingo https://www.youtube.com/channel/UCoo3pkmAU1cmrdumo9SLNUQ/vídeos

Radio UdeG Ocotlán https://www.youtube.com/user/RadioUdeGOcotlan/vídeos

Radio UNAM https://www.youtube.com/channel/UCB_CLh72OkgGSjLjj0uJPng/vídeos

UACJ Radio https://www.youtube.com/user/UACJTV/vídeos

Uni Radio https://www.youtube.com/channel/UCJ9lqTIXzklys94-ez4HFBw/vídeos

Universo 94.9 https://www.youtube.com/user/Universo949/vídeos?disable_polymer=1 